\title{
Min-BASED SYMMetry POSSIBILISTIC NeTWORK MODEL FOR REPRESENTATION OF UNCERTAIN DATA MODELS
}

\author{
K. Madhavi ${ }^{1}$, P. E. S. N. Krishna Prasad ${ }^{2}$, and B. D. C. N. Prasad $^{3}$ \\ ${ }^{1}$ Dadi Institute of Engineering and Technology, Anakapalli, India \\ ${ }^{1}$ kolukulurimadhavi@yahoo.com \\ ${ }^{2} \& 3$ Prasad V. Potluri Siddhartha Institute of Technology, India \\ ${ }^{2}$ surya125@gmail.com and ${ }^{3}$ bdenprasadegmail.com
}

\begin{abstract}
Uncertainty is inherent in various applications, such as Sensor Networks, Large Datasets, Medicine, Mobile Networks, Biomedical and Clinical Data, Social and Economical Research. Uncertain data poses significant challenges for data analytic tasks. Analysis of large collections of uncertain data is a primary task in these applications, because data is vague, ambiguous, incomplete, and inefficient. In this paper, we investigate the fundamental problem of analysis and representation of uncertain data objects for processing. Representation of uncertain data in various approaches such as Probabilistic based, Possibilistic based, plausibility based theory and so on, in terms of Data Streams, Linkage models, DAG models, etc. Among these Possibilistic data models are the most simple, natural way to process and produce the optimized results through Query processing. In this paper, we propose the Uncertain Data model can be represented as a Min-based symmetry Possibilistic data model and vice versa using linkage data model through possible Worlds.
\end{abstract}

\section{KEYWORDS}

Uncertain Data; Uncertain Object model; Possibilistic Data model; Possibilistic Linkages; Possibilistic Linkage model; Possible worlds; Min-based symmetry Operator;

\section{INTRODUCTION}

The construction of mathematical theory of risk has been realized in the framework of possibility theory. Possibilistic methods constitute a very efficient tool in the analysis of uncertain data. The evaluations and forecasting's realized with Possibilistic models are efficient for the large data. Possibility theory initiated by Zadeh [32 ] is an alternative to probability theory in the treatment of uncertainty. It studies those situations of uncertainty in which the events do not occur a large number of times and therefore the information is not extracted from a large volume of data. It has been successfully applied in decision making problems in conditions of uncertainty.

Possibility theory is based on new concepts such as possibility measure, necessity measure, possibility distributions, etc. Traditionally, possibilistic distributions are interpreted as fuzzy sets. The notions of probability and possibility express different aspects of uncertainty. Probability theory offers a quantitative model for randomness and indecisiveness, possibility theory offers a qualitative model of incomplete knowledge [32]. 
Possibility theory $[4,19,33]$ offers a natural and simple model to handle uncertain data. It is a framework for experts to express the uncertainty numerically in terms of possibility degrees in the universe of discourse. The Propositions of product based and min based possibilistic data models discussed in [1,4 and 5]. The aim of this paper presents to represent the uncertain data model into min-based symmetry possibilistic data model and vice versa using possible worlds.

\section{Min BaSed Possiblistic ApProach}

The Possibility theory offers two approaches of conditioning 1) min-based and 2) product based operators. This leads to these two possible approaches of directed casual possiblistic networks. The product based possibilistic network model for representing uncertain data discussed in[1]. The min-based approach to represent uncertain will be discussed in the further sections.

\subsection{Conditional Independence relations}

Conditional independence[4,5] relations between variables play an important role in handling uncertain data in possibilistic approach. There two ways conditional independence relations can be described.

1. Decompositional independence ensures the decomposition of a joint distribution pertaining to tuples of variables into local distributions on smaller subsets of variables.

2. Causal independence, expressing the lack of causality between variables. This kind of independence is characterized in semantic representations. For instance, a variable is said to have no influence on another variable, if our belief in the value of the latter does not change when learning something about the value of the former.

From the observations[4,5,17 and 23] of these two independence relations, decompositional independence is recommended for the representation of uncertain data models.

\subsection{Independence Relations in Possibilistic Framework}

From [3,31,33], two types of independence relations have been considered such as 1) Possibilistic Casual Independence 2) Possibilistic Decompositional Independence: Non-Interactivity. For these two independence relations, possibility theory has several kinds of conditioning relations such as 1) Plausibility independent 2) min-based independent 3) Product-based independent 4) Pareto Independent 5) Leximin independent 6) Leximax independent relations. These relations have been discussed in [3,31,33]. From the observation of [3,31], min-based independence decomposition is considered in the possibilistic decompositional independence relation with the help of Graphical models. Basically a graphical model supports 5 properties such as P1: Symmetry, P2: Decomposition, P3: Weak Union, P4: Contraction, P5: Intersection.

The independence relations supports only few of the properties[3] which are mentioned above, listed in Table2. 
International Journal of Computer Science, Engineering and Applications (IJCSEA) Vol.2, No.6, December 2012

Table2: Graphical Properties on Independence Relations

\begin{tabular}{|l|c|c|c|c|c|}
\hline $\begin{array}{c}\text { Independence } \\
\text { Relation }\end{array}$ & $\begin{array}{c}\text { Symme } \\
\text { try }\end{array}$ & $\begin{array}{c}\text { Decomp } \\
\text { osition }\end{array}$ & $\begin{array}{c}\text { Weak } \\
\text { Union }\end{array}$ & $\begin{array}{c}\text { Contr } \\
\text { action }\end{array}$ & $\begin{array}{c}\text { Intersecti } \\
\text { on }\end{array}$ \\
\hline Non-Interactivity & yes & yes & yes & yes & no \\
\hline Min-based & no & yes & yes & yes & yes \\
\hline $\begin{array}{l}\text { Min-based } \\
\text { Symmetry }\end{array}$ & yes & yes & yes & yes & yes \\
\hline Product-based & yes & yes & yes & yes & yes if $\pi>0$ \\
\hline Pareto & yes & yes & yes & yes & yes \\
\hline Plausibility & no & yes & yes & yes & yes \\
\hline Leximax & yes & yes & no & no & no \\
\hline Leximin & yes & yes & no & no & no \\
\hline
\end{tabular}

From these observations, the casual and decompositional independence relations based on product and $\mathrm{min}$ operators are reasonable relations with good properties, since they are semigraphiods. Indeed, the min-based with symmetry property independence relation is too strong than the rest of the relations, $[3,14]$, this relation has been considered in the Possibilistic Graphical model. The product-based relation is good but it cannot satisfy the intersection property, it is good and most used operator in the Probabilistic network models. It is good and most used operator, the min-based independence relation is considered to represent uncertain data model using Possibilistic graphical models.

\subsection{Min-based Possibilistic Networks}

A min-based possibilistic graph[3,19,31] over a set of variables, denoted by $\boldsymbol{P o s s} \boldsymbol{G}_{\boldsymbol{m}}$, is a possibilistic graph, where conditionals are defined using min-based conditioning :

$$
\operatorname{Poss}\left(\left.\omega\right|_{m}, \varphi\right)=\pi\left(\left.\omega\right|_{m}, \varphi\right)=\left\{\begin{array}{cc}
1 & \text { if } \pi(\omega)=\Pi(\varphi) \text { and } \omega \in \varphi \\
\pi(\omega) & \text { if } \pi(\omega)<\Pi(\varphi) \text { and } \omega \in \varphi \\
0 & \text { otherwise }
\end{array}\right.
$$

The joint distribution relative to min-based possibilistic networks can be computed using the minbased chain rule.

Min-based chain rule: Given a min-based possibilistic network $G_{m}$, the global joint possibility distribution over a set of variables $V=\left\{A_{1}, A_{2}, \ldots . ., A_{n}\right\}$ can be expressed as the minimum of the $\mathrm{N}$ initial a Priori and conditional possibilities via min-based chain rule: $\operatorname{Poss}_{m}\left(A_{1}, A_{2}, \ldots . ., A_{n}\right)=\min _{i=1 \ldots N} \prod\left(A_{i} \mid U_{i}\right)$, which is derived from the minimum independence relations. From the non-interactivity of the min-based is defined by $\Pi(x \wedge y \mid z)=\min (\Pi(X \mid z), \Pi(y \mid z)), \forall x, y, z$ to express that the variables sets $\mathrm{X}$ and $\mathrm{Y}$ are non-interactivity independent in the context of $Z$.

Let $d=\left\{A_{1}, A_{2}, \ldots . . . A_{N}\right\} \quad$ be an ordering of the variables in $\mathrm{V}$ such that $\forall A_{i}, U_{A_{i}} \subseteq\left\{A_{i+1}, \ldots . ., A_{N}\right\}$. Let $R_{A_{i}}=V-U_{A_{i}}$. Then by the definition of min-based chain rule: 
International Journal of Computer Science, Engineering and Applications (IJCSEA) Vol.2, No.6, December 2012

$$
\begin{aligned}
\pi_{\text {min }}\left(A_{1}, A_{2}, \ldots . A_{N}\right) & =\min \left(\Pi\left(A_{1}, R_{A_{1}} \mid U_{A_{1}}\right), \Pi\left(U_{A_{1}}\right)\right) \\
= & \min \left(\Pi\left(A_{1}, U_{A_{1}}\right), \Pi\left(A_{2}, A_{3}, \ldots . ., A_{N}\right)\right)
\end{aligned}
$$

It may be noted that, in the min-based Possibilistic networks do not recover the initial data provided by the experts, since the unrecovered data correspond to the redundant data that can be ignored and have no influence on independence relations.

\section{UnCERTAIN DATA MODEL}

Modeling and Querying uncertain data $[7,15,21,23,25]$ has been a fast growing research direction and receives an increasing attention. Various models of uncertain and fuzzy data have been developed. We proposed a novel model for modeling uncertain data in the fuzzy environment using Possibilistic data model. The working model for uncertain data describes the existence possibility of a tuple in an uncertain data set and the constraints on the uncertain tuples.

A fuzzy database $[11,12,15,19]$ comprises of multiple fuzzy tables. A fuzzy table contains a set of tuples, where each tuple is associated with a fuzzy membership value, which is treated as Degree of Possibility in the Possibilistic Model. A fuzzy table may also come with some generation rules to capture the dependencies among tuples, where a generation rule specifies a set of exclusive tuples, and each tuple is involved in at most one generation rule.

Another useful model is the Uncertain Object Model [7,10,19]; an uncertain object is conceptually described by a fuzzy membership function, i.e. Possibility Distribution in the data space. In this scenario a possibility degree of an uncertain object is unknown, a set of samples (instances) are collected to approximate the fuzzy distribution, which is a possibility distribution.

Definition: An Uncertain Object is a set of instances $U=\left\{u_{i}, u_{2}, \ldots \ldots . u_{n}\right\}$ such that each instance $u_{i}(1 \leq i \leq n)$ takes a possibility membership $\operatorname{Poss}\left(u_{i}\right)=\pi\left(u_{i}\right)>0$ and $\sum_{i=1}^{n} \pi\left(u_{i}\right)=1$.

The cardinality of an uncertain object $U=\left\{u_{i}, u_{2}, \ldots \ldots . u_{n}\right\}$ denoted by $|\mathrm{U}|$ is the number of instances contained in $\mathrm{U}$. The set of all uncertain objects denoted by $\mathrm{X}, X=\left\{U_{1}, U_{2}, \ldots \ldots . U_{n}\right\}$.

\subsection{Possible worlds of Uncertain Objects}

A possible world is a full description of how the history of the universe might have gone that is logically possible. The laws of logic set the only constraint on possibility in this wide inclusive sense. A possible world might include events that are physically impossible, not consistent with the fundamental laws of nature. A possible world is a possible way the world (the real world or some imaginary world) could be. For instance, when representing a crossword puzzle, the possible worlds correspond to the ways the crossword could be filled out. Possible worlds are described by algebraic variables. An algebraic variable is a symbol used to denote features of possible worlds. Possible worlds can be defined in terms of variables or variables can be defined in terms of possible worlds.

Definition 3.2: Possible worlds of Uncertain Objects can be defined as: Let $U=\left\{u_{i}, u_{2}, \ldots \ldots . . u_{n}\right\}$ be a set of uncertain objects. A Possible world $W=\left\{w_{i}, w_{2}, \ldots \ldots w_{m}\right\},\left(w_{i} \in U_{i}\right)$ is a set of instances such that one instance is taken from each uncertain object. The possibility of $W$ (min- 
based) is $\operatorname{Poss}(W)=$ Poss $_{\min }\left(w_{i}\right), 1 \leq i \leq m$, where $W$ is the set of all possible worlds. The number of all possible worlds is $|W|=\operatorname{Poss}_{\min }\left|w_{i}\right|$ and $\operatorname{Poss}(W)=\pi(W)=\sum_{w \in W} \pi_{\min }(w)=1$.

\subsection{Possibilistic Table}

A Possibilistic database model is used to represent uncertain data, which is a finite set of Possibilistic tables; A Possibilistic table contains a set of uncertain tuples $\mathrm{T}$ and a set of generation rules $\mathfrak{K}$. Each uncertain tuple $t \in \mathrm{T}$ is associated with a possibility degree, $\operatorname{Poss}(t)=\pi(t)>0$. Each generation rule $\mathrm{R} \in \mathfrak{K}$ specifies a set of exclusive tuples in the form $R: t_{r_{1}} \oplus t_{r_{2}} \oplus t_{r_{3}} \oplus \ldots \ldots . . t_{r_{m}}$, where $t_{r_{i}} \in T(1 \leq i \leq m)$

$$
\operatorname{Poss}\left(t_{r_{i}} \wedge t_{r_{j}}\right)=0,(1 \leq i, j \leq m, i \neq j) \text { and } \sum_{i=1}^{m} \operatorname{Poss}\left(t_{r_{i}}\right)=1
$$

The cardinality of a generation rule $\mathrm{R}$, denoted by $|\mathrm{R}|$, is the number of tuples involved in $\mathrm{R}$. The generation rule $\mathrm{R}$ is the set of all tuples $t_{r_{1}}, t_{r_{2}}, t_{r_{3}} \ldots . ., t_{r_{m}}$ involved in the rule, at most one tuple can appear in a possible world. $\mathrm{R}$ is a singleton rule if there is only one tuple involved in the rule, otherwise $\mathrm{R}$ is a multiple rule, and thus the Possibilistic database follows a possible world.

Given a Possibilistic Table $\tilde{T}$ a possible world $\mathrm{W}$ is a subset of $\tilde{T}$ such that for each generation rule $R \in \mathfrak{R}_{T},|R \cap W|=1$ if $\operatorname{Poss}(R)=1$, and $|R \cap W| \leq 1$ if $\operatorname{Poss}(R)<1$, Thus, the existing membership of $\mathrm{W}$ is

$$
\operatorname{Poss}(W)=\prod_{R \in \mathfrak{R}_{T},|R \cap W|=1} \operatorname{Poss}_{\min }(R \cap W) \prod_{R \in \mathfrak{R}_{T},|R \cap W|=\phi}\left(1-\operatorname{Poss}_{\min }(R)\right)
$$

For an uncertain table $\tilde{T}$ with a set of generation rules $\Re_{T}$, the number of all possible worlds is

$$
|W|=\prod_{R \in \mathfrak{R}_{T}, P \text { Ooss }(R)=1}|R| \prod_{R \in \mathfrak{R}_{T}, P \text { Ooss }(R)<1}|R|+1
$$

We can convert the Uncertain Object model into Possibilistic database model $[10,25,28,30]$ and vice versa. When, it is concerned that both are equivalent. The conversion process will be presented below.

1. Conversion between Uncertain Object Model to Possibilistic Database Model: A set of uncertain objects can be represented by a fuzzy table. For each instance ' $x$ ', of an uncertain Object ' $\mathrm{X}$ ', to create a tuple $t_{x}$, whose membership or possibility degree value is $\operatorname{Poss}\left(t_{x}\right)=\pi_{\min }\left(t_{x}\right)$ for each uncertain object $X=\left\{x_{1}, x_{2}, \ldots \ldots . x_{m}\right\}$ to create one generation rule $R_{X}: t_{x_{1}} \oplus t_{x_{2}} \oplus \ldots . . \oplus t_{x_{m}}$.

2. Conversion between Possibilistic Database models to Uncertain Object Model: A fuzzy table can be represented by a set of uncertain objects with discrete instances. For each tuple $t$ in a fuzzy table, to create an instance $x_{t}$, whose membership or possibility degree is $f\left(x_{t}\right)=\operatorname{Poss}\left(x_{t}\right)=\pi_{\text {min }}(t)$. For a generation rule $R: t_{r_{1}} \oplus t_{r_{2}} \oplus \ldots \ldots \oplus t_{r_{m}}$, to create an uncertain object $X_{R}$, which includes instances $x_{t_{x_{1}}} \oplus x_{t_{x_{2}}} \oplus \ldots \ldots \oplus x_{t_{x_{m}}}$ corresponding to 
$t_{r_{1}}, t_{r_{2}}, \ldots \ldots, t_{r_{m}}$, respectively. Moreever, $\sum_{i=1}^{m} \operatorname{Poss}\left(t_{r_{i}}\right)=\sum_{i=1}^{m} \pi_{\min }\left(t_{r_{i}}\right)<1$. We create another instance $x_{Q}$ whose fuzzy membership function is $f\left(x_{Q}\right)=1-\sum_{i=1}^{m} \pi_{\min }\left(t_{r_{i}}\right)$ and $u_{Q}$ to the uncertain object $X_{R}$.

\section{Possibilistic LinKage Model}

In the basic uncertain object model, we assume that each instance belongs to a unique object, though the object may have multiple instances, if an instance may belong to different objects in different possible worlds. Such a model is useful in Possibility Linkage analysis.

A Possibilistic linkage model[10,15,16,19] contains two sets of tuples A and B and a set of linkages $\mathfrak{I}$. Each linkage $\boldsymbol{\ell}$ in matches one tuple in A and one tuple in B. For a linkage $\boldsymbol{\ell}$ $=\left(t_{A}, t_{B}\right)$, we say $\boldsymbol{\ell}$ is associated with $t_{A}$ and $t_{B}$. We write $\boldsymbol{\ell} \in t_{A}$ and $\boldsymbol{\ell} \in t_{B}$. We consider each tuple $t_{A} \in A$ as an uncertain object and $t_{A} \in B$ as an instance of $t_{A}$ if there is a linkage $\boldsymbol{\ell}=\left(t_{A}, t_{B}\right) \in \mathfrak{T}$. The membership possibility of instance $t_{B}$ with respect to object $t_{A}$ is $\pi(\boldsymbol{\ell})$, which is $\mu(\boldsymbol{\ell})$.

Object $t_{A}$ may contain multiple instances $\left\{t_{B_{1}}, t_{B_{2}}, \ldots \ldots, t_{B_{k}}\right\}$ where $\left(t_{A}, t_{B_{i}}\right) \in \mathfrak{I}(1 \leq i \leq k)$. At the same time, an instance $t_{B}$ may belong to multiple objects $\left\{t_{A_{1}}, t_{A_{2}}, \ldots . . ., t_{A_{d}}\right\}$ where $\left(t_{A_{j}}, t_{B}\right) \in \mathfrak{I}(1 \leq j \leq d)$. A mutual exclusion rule $R_{T_{B}}=\left(t_{A_{1}}, t_{B}\right) \oplus\left(t_{A_{2}}, t_{B}\right) \oplus \ldots \ldots,\left(t_{A_{d}}, t_{B}\right)$ specifies that $t_{B}$ can only belong to one object in a possible world.

A record linkage $[7,11,26]$ is a technique that finds the linkages among data entries referring to the same real world entities from different data sources. In the real world applications, data is often incomplete or ambiguous. Thus, record linkages are often uncertain.

Possibility Record Linkages [7,11,26,29]are often used to model the uncertainty. For teo records, a state- of-the art, possibility record linkage model can estimate the possibility degree that the two records refer to the same real world entity. Let us consider teo thresholds $\alpha_{1} \& \alpha_{2}\left(0 \leq \alpha_{1}<\alpha_{2} \leq 1\right)$. When the possibility linkage is less than $\alpha_{1}$, the records are not matched. When the possibility linkages are between $\alpha_{1} \& \alpha_{2}$, then records considered possibly matched.

To build a possibility record linkage effectively and efficiently with the some real world scenarios. Each linked pair of records as an uncertain instance and each record as an uncertain object. Two uncertain objects from different data sets may share zero or one instance. Thus the uncertain objects may not be independent. For instance, let us consider the patient data from hospitalized registered and cause of death data, which is presented in Table3. 
International Journal of Computer Science, Engineering and Applications (IJCSEA) Vol.2, No.6, December 2012

Table3: Record linkages between the patients registered data and cause of death registered data.

\begin{tabular}{|c|c|c|c|c|c|c|c|}
\hline \multirow{2}{*}{$\begin{array}{l}\text { Lin } \\
\text { kag } \\
\text { e ID }\end{array}$} & \multicolumn{3}{|c|}{ Patient Registered Data } & \multicolumn{3}{|c|}{ Cause of Death Data } & \multirow{2}{*}{$\begin{array}{c}\text { Possib } \\
\text { ility } \\
\text { Degre } \\
\text { e }\end{array}$} \\
\hline & $\begin{array}{l}\text { PI } \\
\text { D }\end{array}$ & $\begin{array}{c}\text { Name of the } \\
\text { Patient }\end{array}$ & Disease & $\begin{array}{l}\text { DI } \\
\text { D }\end{array}$ & $\begin{array}{l}\text { Name of the } \\
\text { Patient }\end{array}$ & Age & \\
\hline$l 1$ & $x 1$ & Sita M. Lakshmi & Flu & $y 1$ & Maha Lakshmi & 42 & 0.4 \\
\hline$l 2$ & $x 1$ & Sita M. Lakshmi & Flu & $y 2$ & M. Lakshmi & 45 & 0.4 \\
\hline 13 & $x 1$ & Sita M. Lakshmi & Flu & $y 3$ & S. Lakshmi & 32 & 0.5 \\
\hline 14 & $x 2$ & S. MahaLakshmi & Cancer & $y 3$ & S. Lakshmi & 32 & 0.2 \\
\hline 15 & $x 2$ & S. MahaLakshmi & Cancer & $y 4$ & S. M. Lakshmi & 55 & 0.8 \\
\hline
\end{tabular}

Let $\mathrm{E}$ be the set of real world entities. Let us consider two tables $\mathrm{A}$ and $\mathrm{B}$ which describe subsets $E_{A}, E_{B} \subseteq E$ of entities in $E$. Each entity is described by at most one tuple in each table. In general, $E_{A}$ and $E_{B}$ may not be identical, they may have different schemas as well.

Possibility Linkage: Consider two tables A and B each describing a subset of entities in E, a linkage function $L: A \times B \rightarrow[0,1]$ gives a score $L\left(t_{A}, t_{B}\right)$ for a pair of tuples $t_{A} \in A, t_{B} \in B$ to measure the likelihood that $t_{A} a n d t_{B}$ describes the same entity in $E$.

A pair of tuples $l=\left(t_{A}, t_{B}\right)$ is called a possibility record linkage, if $L(l)>0, \operatorname{Poss}(l)=L\left(t_{A}, t_{B}\right)$ is the possibility degree of ' $l$ '. Given a linkage $l=\left(t_{A}, t_{B}\right)$, the larger the possibility degree $\operatorname{Poss}(l)$, the more likely the two tuples $t_{A} a n d t_{B}$ describe the similarity entity.

A tuple $t_{A} \in A$ may participate in zero, one or multiple linkages. The number of linkages that $t_{A}$ participates in as called the Degree of $t_{A}$ denoted by $d\left(t_{A}\right)$. Similarly we can define $d\left(t_{B}\right)$.

For a tuple $t_{A} \in A$, let $l_{1}=\left(t_{A}, t_{B_{1}}\right), \ldots \ldots \ldots . ., l_{d}=\left(t_{A}, t_{B_{d}}\right)$ be the linkages that $t_{A}$ participates in. For each tuple $t_{A} \in A$, we can write a Mutual Exclusive Rule (MER) $R_{t_{A}}=l_{1} \oplus l_{2} \oplus \ldots \ldots . \oplus l_{d\left(t_{A}\right)}$, where $d$ is the degree of $t_{A} \in A$, that indicates atmost one linkage can hold based on the assumption that each entity can be described by atmost one tuple in each table. The possibility degree is computed as $\operatorname{Poss}\left(t_{A}\right)=\sum_{i=1}^{d\left(t_{A}\right)} \operatorname{Poss}\left(l_{i}\right)$ that $t_{A}$ is matched by some tuples in B. Since the linkage function is normalized, $\operatorname{Poss}\left(t_{A}\right) \leq 1$. It is denoted by $R_{A}=\left\{R_{t_{A}} \mid t_{A} \in A\right\}$, the set of mutual exclusion rules for tuples in A. Similarly $R_{t_{B}}$ for $t_{B} \in B$, are symmetrically defined.

Therefore $(£, A, B)$ specifies a bipartite Graph, where tuples in $\mathrm{A}$ and those in $\mathrm{B}$ are two independent sets of nodes respectively and the edges are the linkages between the tuples in the two data tables. 
International Journal of Computer Science, Engineering and Applications (IJCSEA) Vol.2, No.6, December 2012

\subsection{Connection with the Uncertain Object Model.}

Given a set of Possibility linkages, $L$ between tuple sets, $A$ and $B$, we consider each tuple $t_{A} \in A$, as an uncertain object. For any tuple $t_{B} \in B$, if there is a linkage $l=\left(t_{A}, t_{B}\right)$ such that $\operatorname{Poss}(l)>0$. Then $t_{B}$ can be considered as an instance of object $t_{A} \in A$ whose possibility degree is $\operatorname{Poss}(l)$.

In contrast to the basic uncertain object model where each instance only belongs to one object, in the Possibility Linkage model, a tuple $t_{B} \in B$ may be the instance of multiple objects $\left\{t_{A_{1}}, t_{A_{2}}, \ldots \ldots, t_{A_{d}}\right\}$ where $\mathrm{d}$ is the degree and $t_{A_{i}}$ is a tuple in $\mathrm{A}$ with linkage $\left(t_{A_{i}}, t_{B}\right) \in L(1 \leq i \leq d)$ A mutual exclusion rule $R_{t_{B}}=\left(t_{A_{i}}, t_{B}\right) \oplus \ldots \ldots \oplus\left(t_{A_{d}}, t_{B}\right)$ specifies that $t_{B}$ should only belong to one object in a possible world.

Alternatively, we consider each tuple $t_{B} \in B$ as an uncertain object and a tuple $t_{A} \in A$ is an instance of $t_{B}$ if there is a linkage $\left(t_{A}, t_{B}\right) \in L$. Thus, a linkage function can be regarded as the summarization of a set of possible worlds.

For a linkage function $L$ and tables $A$ and $B$, let $L_{A, B}$ be the set of linkages between tuples $A$ and B. A Possible world of $L_{A, B}$ denoted by $W \subseteq L_{A, B}$ is a set of pairs $l=\left(t_{A}, t_{B}\right)$ such that

1. For any mutual exclusion rule $R_{t_{A}}$, ifPoss $\left(t_{A}\right)=1$, then there exists one pair $\left(t_{A}, t_{B}\right) \in W$. Symmetrically, for any mutual exclusion rule, $R_{t_{B}}$, ifPoss $\left(t_{B}\right)=1$ then there exists one pair $\left(\left(t_{A}, t_{B}\right) \in W\right.$.

2. Each tuple $t_{A} \in A$ participates in at most one pair in $\mathrm{W}$, so does each tuple $t_{B} \in B$. $W_{L_{A, B}}$ denotes the set of all possible worlds of $L_{A, B}$

3.

Similarly we can represent the uncertain data models in the form of Data Streams as well as Possibilistic Graphical models using Possibilistic Networks that can be discussed in future presentations.

\section{CONCLuSion}

The object of this paper is to represent the uncertain data using Min based Possibilistic object models for processing and evaluating Query and also give the ranking to the evaluated query. Here, an uncertain object model is represented as Possibilistic Database Model using Possibilistic Networks through Min-based operator and vice versa so that the uncertain data model can be evaluated through the query evolution mechanism using Possibilistic Database model. Further, the uncertain data may be represented as Data streams and Possibilistic Graphical Models that process the data objects to evaluate through query evaluation mechanism using Possibility theory. 
International Journal of Computer Science, Engineering and Applications (IJCSEA) Vol.2, No.6, December 2012

\section{REFERENCES}

[1] P. E. S. N. Krishna Prasad, K. Madhavi and B. D. C. N. Prasad, "Representation of Uncertain Data using Possibilistic Network Models”, Sundarapandian et al. (Eds): CoNeCo,WiMo, NLP, CRYPSIS, ICAIT, ICDIP, ITCSE, CS \& IT 07, pp. 267-278, 2012. @ C CS \& IT-CSCP 2012

[2] Christian Borgelt, Learning Possibilistic Graphical Models from Data, IEEE Transactions on Fuzzy Systems, 2003.

[3] Christian Borgelt, J. Gebhardt, and Rudolf Kruse, Learning from Imprecise data: Possibilstic Graphical Models, Journal Computational Statistics \& Data Analysis - Nonlinear methods and data mining, Vol 38, No 4 2002, pp. 449 - 463.

[4] Nahla Ben Amor, Qualitative Possiblistic Graphical Models: From Independence to Propogation algorithms. Ph. D. Thesis, 2012

[5] Salem Benferhat, Didier Dubois Laurent Garcia and Henri Prade, Possibilistic logic bases and Possibilistic Graphs.

[6] Thomos N. Herzog, Fritz J. Scheuren and William E. Winkler, Data Quality and Record Linkages, Springer, 2007,pp.81-92.

[7] Amihai Motro, Imprecision and Uncertainty in Database Systems,

[8] Angelos Vasilakopoulos andVerena Kantere, Efficient Query Computing for Uncertain Possibilistic Databases with Provenance.

[9] Jorg Gebhardt and Rodulf Kruse , Learning Possibilistic Graphical Networks from Data, IEEE Transaction on Fuzzy Systems

[10] Ming Hua, Jian Pei, Ranking Queries on Uncertain data, Advances in Database Systems.

[11] M. S. Sunitha and A. Vijay Kumar, Complement of a Fuzzy Graph, Indian J. Pure Applied Mathematics, 33(9), 1451-1464, September 2002.

[12] Damiani. E, Tanca L.,Building Queries to XML data, Database and Expert Systems Applications, 2000, pp 266-279.

[13] Christian Borgelt, J“org Gebhardt, and Rudolf Kruse, Possibilistic Graphical Models,

[14] Pascale FONCK, Conditional Independence in Possibility Theory, UAI'94 Pp.224 - 226.

[15] Christian Borgelt, and Rudolf Kruse, Data Mining with Possibilistic Graphical Models

[16] Salem Benferhat and Salma Smaoui, A Hybrid Possibilistic Networks, International Journal of Approximate Reasoning, Vol 44, No.3, 2007,224-243.

[17] Salem Benferhat and Salma Smaoui, on the use of Possibistic bases for local computations in product based possibilistic Networks

[18] Christian Borgelt and J“org Gebhardt, A Naïve Bayes style Possibilistc Classifier, Proc. 7th European Congress on Intelligent Techniques and Soft Computing.

[19] Anish Das Sarma, Omar Benjelloun, Alon Halevy,Jennifer Widom, Working Models for Uncertain data, Data Engineering ICDE-2006

[20] Christian Borgelt and Rudolf Kruse, Operations and Evoluation measures for Learning Possibilistic Graphical Models, Artificial Intelligence, Vol. 148, No.1-2, 2003, pp. 385-418.

[21] Jiang Chen and Ke Yi, Dynamic structures for Top-kqueries on Uncertain Data, Proceedings of the 18th international conference on Algorithms and computation, 2007, pp. 427-438

[22] AbdelKader Heni, M Nazih Omri and Adel M Alimi, Knowledge Representation with Possibilstic and Certain Bayesian Networks, Proceedings of the 4th WSEAS Int. Conf. on Information Security, Communications and Computers, Tenerife, Spain, 2005, pp472-477

[23] C.C. Agarwal, Managing and mining Uncertain Data

[24] Brian Babcock Shivnath Babu Mayur Datar Rajeev Motwani Jennifer Widom, Models and Issues in Data Stream Systesms, Springer, 2002 pp1-16.

[25] Anish Das Sarma, Omar Benjelloun, Alon Halevy, Shubha Nabar, and JenniferWidom, Representing Uncertain Data: Models,Properties and Algorithms, VLDB Journal, 2009.

[26] Salem Benferhat and Salma Smaoui, Possibilistic Casual Networks for handling Interventions: A New Propogation Algorithm

[27] Salem Benferhat, Didier Dubois Laurent Garcia and Henri Prade, On the Transformation between Possibilistic Logic bases and Possibilistic Casual Networks, International Journal of Approximate Reasoning 29 (2002) 135-173.

[28] Jiang Chen and Ke Yi , Ranking Distributed Probabilistic Data, ACM SIGMOD-2009, pp:361-374.

[29] Goetz Graefe, Query Evaluation Techniques for Large Databases, ACM Computing Surveys, Vol25,No. 2, 1993, pp. 73-169 
International Journal of Computer Science, Engineering and Applications (IJCSEA) Vol.2, No.6, December 2012

[30] Graham Cormode, Divesh Srivastava, Entong Shen and Ting Yu, Aggregating Query Answering on Possibilistic Data with Cardinality ConstraintsICDE,2012

[31] D. Dubois and H. Prade, Possibility Theory: an approach to Computerized Processing of Uncertainty, Plenum Press, 1988.

[32] Lawrence J. Mazlack, Using Imprecise Complexes to Computationally Recognize Casual Relations in Very Large Data Sets, Proceedings of IPMU, 2008,pp. 698-704

[33] Irina Georgescu, Possibility Theory and the Risk, Studies in Fuzziness and Soft Computing, Springer, 2012

[34] Jirina Vejnarova, Conditional Independence in Evidence Theory, Proceedings of IPMU, 2008, pp.938-945. G. Shafer, Mathematical Theory of evidence, Princston Press, 1976. 